(C)2009 IEEE. Personal use of this material is permitted. However, permission to reprint/republish this material for advertising or promotional purposes or for creating new collective works for resale or redistribution to servers or lists, or to reuse any copyrighted component of this work in other works must be obtained from the IEEE. 


\title{
Information Ecological Imbalance and Information Intervention Policies in Digital Ecosystems
}

\author{
Dengya Zhu ${ }^{1}$, Member, IEEE, Hongchuan Wang ${ }^{2}$, and Elizabeth Chang ${ }^{1}$, Senior Member, IEEE \\ ${ }^{1}$ Dengya Zhu, Heinz Dreher, and Elizabeth Chang, Curtin Business School, Curtin University of Technology, Perth Australia \\ e-mail : dengya.zhu@postgrad.curtin.edu.au, e.chang@curtin.edu.au \\ ${ }^{2}$ Hongchua Wang, Henan Public Security Academy, Zhengzhou, Henan, China \\ e-mail:whc@hngazk.edu.cn
}

\begin{abstract}
This article explores ecological imbalance in digital ecology environment. With the dramatic growth of digital information in the world, organizations, in an age of information ecology can easily be affected by the consequences of information ecological imbalance. This imbalance may be triggered by maladjustment of the circulation in the system, by the abnormal flowing of some kinds of information, or the malfunction of some components in the chain of information flow. An information flow intervention approach, which imposes some control over information flow by means of semantic annotation, is proposed from the perspective of operationality and feasibility. The principal components in the proposed model are explained and the working principle of the model is explained. This research aims to realize ecological balance in a digital ecosystem by intervening in the flow of information, and consequently, providing some mechanism that will lead to the universal governance of the digital ecosystem.
\end{abstract}

Index Terms-digital ecosystems, information ecology, information flow, ecological imbalance, information intervention.

\section{INTRODUCTION}

The sustainability and stability of any ecosystem relies on maintaining the dynamic balance of the system by effective energy flow, material flow, and information flow cycles to assimilate waste and be able to recover from damage should threats appear [1]. Effectiveness of these cycles to maintain a dynamic balance is illuminated by the harmonious characteristics of the natural ecological environment which is a complex system evolved over millions of years [1].

Information ecology ${ }^{1}$, according to [2], is a hybrid science which converges the two concepts of "information revolution" and "green revolution". An information ontology, then could be described as "the sum total of information quality, management, products and value, as well as the evaluation of information services and need and liability" [2].

The extraordinarily large number of existing information systems can be seen in terms of digital ecosystems or information ecosystems. As pointed out by [2], "information ecology is a science which studies the laws governing the influence of information summary on the formation and functioning of bio-systems, including that of individuals, human communities and humanity in general and on the health and psychological, physical and social well-being of

\footnotetext{
${ }^{1}$ We make no differences among the terms 'information ecology', 'information ecosystems', 'digital ecosystems', or 'digital information ecosystems'. A detailed discussion is presented in Section II.
}

the human being; and which undertakes to develop methodologies to improve the information environment". In an information ecosystem, species (people - information producers, messenger, and recipient) interact with contemporary information techniques to exchange digital information with each other in this digital environment. The flow of information, consequently, constitutes a digital information flow cycle. Similar to a healthy natural ecological system, the information flow in a digital ecosystem is in dynamic balance [3], but not in stasis. Ecosystem balance is a state where elements or species are highly adaptive, harmonious and uniform. Each species in the system relates closely with others, depends on them, and co-evolves [4].

However, the equilibrium of a digital ecosystem is disturbed when 1) extreme adjustment occurs in the cycle of information flow; 2) irregular information flow occurs; and 3 ) some components in the chain of information flow malfunction [5]. Digital information pollution, information overload, and information shortages are the main characteristics of information ecological imbalance.

Researchers [3, 5, 6] have studied the issue of information ecological imbalance, and while solutions have been proposed to control, manage and govern information flow in an information ecosystem. Comprehensive research in this field is still needed.

In this paper, an information intervention model is proposed. The model differentiates from other models in that it intends to suppress the sources of information noise by intervening and monitoring the metadata of the information sources, and thus boost the quality the information before it can flow into the information ecosystem. The consequence is to keep the information ecosystem healthy.

The paper is organized as follows. Section II discusses the related research in the field of information flow and information ecology. In section III, a conceptual framework is proposed to deal with some of the issues which have potential influences on digital ecosystem ecological balance. The mechanism of the proposed model is also explained. Section IV discusses several problems which need to be further addressed and possible solutions for these problems. Lastly, section $\mathrm{V}$ is our conclusion.

\section{RELATED WORK}

An ecosystem is a self-sustaining system where each species benefits from each others' participation via energy exchange, substance metabolism, and information transfer [4]. We will first review research into digital ecosystems, and then concentrate on digital information flow. 
Before moving on, several concepts are defined first.

According to Webster's Online Dictionary (www.websters-online-dictionary.org/), the definition of data, information, and knowledge are:

Data: A collection of facts from which conclusions may be drawn.

Information: 1. A message received and understood that reduces the recipient's uncertainty. 2. A collection of facts from which conclusions may be drawn.

Knowledge: The psychological result of perception and learning and reasoning.

\section{A. Information Ecology}

The concept of information ecology has been examined by scholars from different points of view.

Davenport and Prusak [7] propose an information ecology model embedded in an organization environment, which is in turn, embedded in the external environment, as shown in Fig. 1. Information ecology is defined as "holistic management of information". Elements - staff, culture/behaviour, politics, architecture, process and strategy in an information environment - interact with each other and keep in balance in the environment.

Effective management of information in the ecosystem involves four attributes. The first attribute is "integration of diverse types of information". This is not limited to hardware, software, and network, but also organizational contextual factors such interpersonal communication, contexts, and emotions. Information ecosystems also need to be flexible enough to evolve with other organisms in external environment; otherwise, the system will be a stumbling block of business excellence. This leads to the second attribute, "recognition of evolutionary change". The third principle concerns "emphasis on observation and description" rather than depiction based on technical architecture and engineering drawings. The last attribute, "focus of people and information behavior", emphasizes the importance of people in the information ecosystem. People are more sensitive to any change in the ecology and will be affected more than other organisms [7]. While these factors are organization oriented; however, inter-organizational behaviours are not well explained in this model.

Nardi and O'Day 's model concentrates on social phenomena and tries to explore the factors behind the phenom-

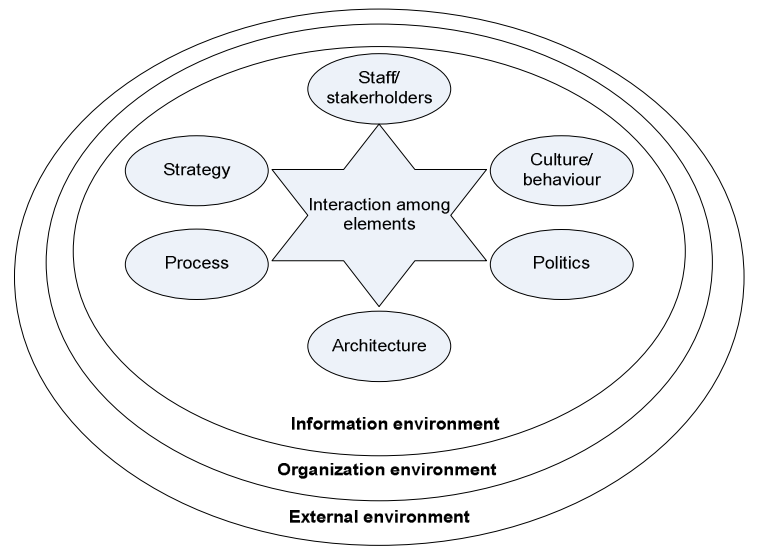

Fig.1 Adaptation of Davenport and Prusak information ecology framework ena. They define information ecology as "a system of people, practices, values, and technologies in a particular local environment" [8]. In the Nardi and O'Day model, information ecology is a system of interrelated people and tools; it contains a diversity of roles for the people and functions for the tools. Another feature of their understanding of information ecology is that there is coevolution over time as new technologies arrive and are assimilated, and as people's roles develop and change; there is a keystone species that is essential to the success of the ecology [8].

Eryomin [2] postulates "Information ecology is a science which studies the laws governing the influence of information summary on the formation and functioning of biosystems, including that of individuals, human communities and humanity in general; on the health and psychological physical and social well-being of the human being; and which undertakes to develop methodologies to improve the information environment" [2].

Baker and Bowker [9] state that an information ecology system "provides a conceptual framework to consider data, the creation of knowledge, and the flow of information within a multidimensional context" [9]. This model emphasizes information management and takes into account information the ecosystem features of complexity, ambiguity, and nonlinearity. The authors propose a three-component conceptual information ecology framework as shown in Fig. 2. Data systems, which is composed of a project structure of data centres, the location where data are received. In the community (learning centres), information flowing from diverse sources intersects and evolves. Thirdly, global networks provide a standard infrastructure for a variety of knowledge grids and for archival purpose.

Malhotra suggests "information ecology is an organization's information environment, and consists of the numerous interacting and interdependent social, cultural, and political subsystems that shape the creation, flow and use of information in the organization" [10]. The proposed knowledge ecology framework emphasizes the feature of "action, performance and adaptation of self-regulatory systems" [10].

Inter-organizational information ecology is defined by Fedorowicz et al. as "a system of people, processes, technologies, and information sharing behaviours, in a local environment, and characterized by colonial processes of adaptation" [11]. This definition extends Davenport and Prusak's model which focuses on the information ecology environment to include inter-organizational environments.

Martin [12] indicates that "information ecology concep-

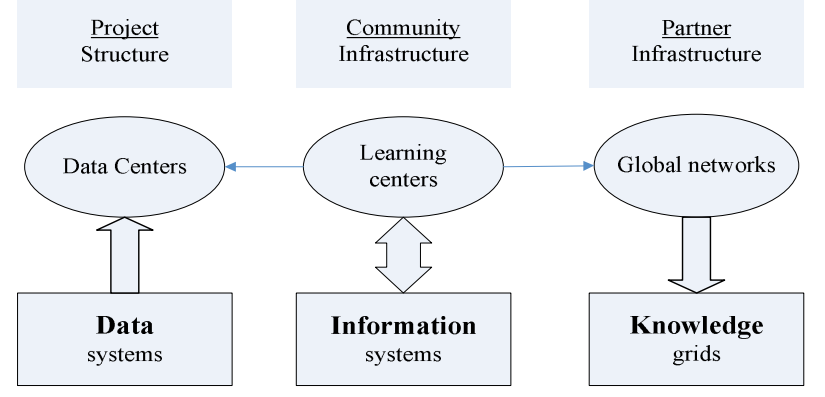

Fig.2 Adaptation of Baker and Bowker information ecology framework 
tualizes the organizational environment as another ecosystem where related systems are coexisting and evolving" [12]. He argues that adaptation is essential for ecosystems to keep the ecological balance in an organizational environment. It is essential not to separate information from the people, facilities, technology and more important, from the context where the information is generated. After analyzing the four attributes of Davenport and Prusak [7], Martin believes Cobit (Control Objectives for Information and related Technology) from the perspective of practical, treats information as the outcomes of an integrated application of "IT related resources that need to be managed by IT process".

A Digital Ecosystem (DES), according to Boley and Chang [13], is "an open, loosely coupled, domain clustered, demand-driven, self-organising agent environment, where each agent of each species is proactive and responsive regarding its own benefit/profit but is also responsible to its system"[13]. From the point of view of ecology, the digital ecosystem is dynamic in that it has the ability to adjust.

An integrated information retrieval model for digital ecosystems is suggested by Zhu and Dreher [14]. This framework combines search results from the intranet, commercial databases, and the Internet to satisfy the information needs of digital ecosystems' users by providing comprehensive and accurate search results. User search preferences, predefined security rules, and other personal information are collected to form a user profile; this profile is then used to refine the search results from different information sources. This model intends to improve the precision of searches, and pays less attention to information flow in digital ecosystems.

In summary, information ecology is an open environment with its ultimate aim to provide effective and efficient information services. Species/organisms in the environment co-evolve harmoniously through exchange of data (a collection of facts), information (facts from which meaning is derived), and knowledge (an outcome of deriving meaning from facts). Organisms are influenced by the environment they live in; and the environment is influenced by the organisms that survive in it.

\section{B. Information flow}

Information flow, intuitively, is the movement of information objects from point of origin to 'target' use. In the process, "simple objects may combine to generate complex objects; the object is modified along the way, maybe used at different points in the flow; and there is no end user" [15].

Barwise and Seligman [16] suggest there are four rules for information flow. The first principle is that information flow results from regularities in a distributed system. Distributed means that information flow can be divided into parts, the flow is from one part (or parts) to another. The presence of regularities links the different parts of the distributed system. The second principle is that information flow crucially involves both types and its particulars. Particulars, or instances are things in the world, which carry information; the information they carry is in the form of types. For example, a may carries information about the mountain. "The map's being of a certain type carries infor- mation that the mountain was of some other type." The third principle concerns the regularities among connections that information about some components of a distributed system carries information about other components. The fourth principle is the regularities of a given distributed system are relative to its analysis in terms of information channels.

In the field of computer security, according to Lowe [17], information flow, in its simplest form, considers two users $\mathrm{H}$ and $\mathrm{L}$ interacting with the same computer system, and asks if there is any flow of information from $\mathrm{H}$ to $\mathrm{L}$; or, can H's behaviour influences L's view of the system? It is then suggested information flow is "the behaviour of one agent can have some influence upon another agent's view of system" [17]. In computer security field, this proposition appears to hold true.

Dretske [18] believes that the origin of information flow is causality. Information flow is a process that is based upon the causal inter-relatedness of source of receiver. A piece of message is conveyed/transmitted from a source to a target by a casual process; what happens at target determines to what happens at source, and vice versa.

A fundamental principle of information flow proposed by Dretske is the Xerox principle which states that "If A carries the information $\mathrm{B}$, and $\mathrm{B}$ carries the information that C, then A carries the information that C" [18]. For example, if a stream of photons carries the information that the light is on, and the light's being on carries the information that the switch is closed, then the stream of photons carries the information that the switch is closed [18]. Dretske's research intends to utilize mathematical theory of communication to develop a theory of information flow, and is theoretical oriented.

An information chain model is proposed by Heeks [19] as shown in Fig. 3. In this model, data are first accessed by an information user; the accessed data are then assessed and selected; the evaluated information is ready for use (application); and finally, there is a reaction to the information. This model emphasizes turning raw data into useful information. However, the model seems too simple, in that it does not differentiate between information consumers and producers.

The information flow model can also be considered from intra-organizational and inter-organizational perspective [20]. Intra-organization information flow concentrates on information processing. The evaluation features of the processing include speed, capture ability, relevant information selection, assessment, and control ability. Inter-organization information flow is concerned with how information flow is distributed outside of the organization. The evaluation features are speed (how fast the information distributes among organizations), capacity, scope, accuracy, error rate, and the reliability of information [20].

There are always some constraints on information flow. Yang [20] indicates that there are institutional and technological constraints. Intellectual property protection, organizational structure, information privilege, culture and cir-

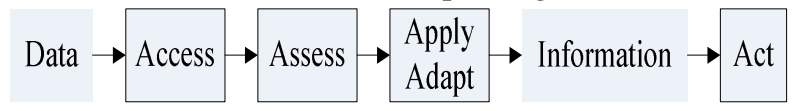

Fig.3 Adaptation of Heeks 4A's information flow model [19] 
cumstance limitations are institutional restrictions. Technological restrictions, that is, information access ability, include information/communication technology infrastructure, mastering of information technology skills of information receiver, and the ability of research and development which makes the access of information easier [20]. All the constraints should be addressed when the information flow model is designed.

\section{INFORMATION INTERVENTION FRAMEWORK}

\section{A. An information intervention model}

In digital ecosystems, information flow occurs at two different levels - intra-organizational and interorganizational. At the intra-organization level, each organism is an information consumer, and at the same time, an information producer. The produced information, when required by other consumers, will flow to its target destination. In this scenario, the information flow is confined within the intranet of a given digital ecosystem. Information flowing within the system is confidential, and is shared only within the system. On the Internet - the inter-organizational environment, the system itself becomes an organism of the World Wide Web, and all the information becomes publicly available. Conceptually, this model is similar to the one proposed by Davenport and Prusak [7] because both models extends from organization to external environment.

One main reason of an information flow ecological imbalance is the information flow pollution caused by information organisms. Another reason is information overload which makes relevant information hard to obtain. We therefore propose an information flow intervention model to address the above two issues. We suggest the pollution can be suppressed within the intra-organizational information flow cycle by means of specification, selection, and filtering.

The proposed model consists of three main parts: organi- zation information sources, personal information sources, and information retrieval.

\section{B. Components in the Model}

\section{1) Organization information sources}

Organization information is selected and filtered before it is allowed to be published. Digital information is annotated by semantic information, which describes the features of the information items to be published. The attributes or metadata tags include title, subject, content description, resource type, source of information, relationship, coverage, author (creator), publisher, contributor, rights, date, format, identifier, language, and usage history. As indicated by [21], meta data are content dependent, and it is very difficult to implement automatic annotation, we therefore prefer to let the information publishers manually perform semantic annotation at the present research stage.

Following [21], the indexing here means key concepts indexing which is different from the traditional word indexing in the field of information retrieval. When indexing, some rules need to be followed. 1) The keywords selected for representing a document should express the subject that is being treated in the document; 2) the keywords selected for the indexing record of a document should name the subjects that are most heavily treated in the document; and 3) the keywords selected for the documents should maximize the probability of retrieving the document [22]. The tf-idf strategy [22] is the most widely accepted indexing weighting strategy, and is suitable here.

tf-idf weighting consists of three components: term frequency $\mathrm{tf}$, inverse document frequency idf, and normalization factor. tf measures the frequency of occurrence of terms in a document or in search terms. idf is document dependent that favours terms concentrated in a few documents in a document collection. This factor varies inversely with the number of documents $\mathrm{n}$ to which a term appearing in a col-

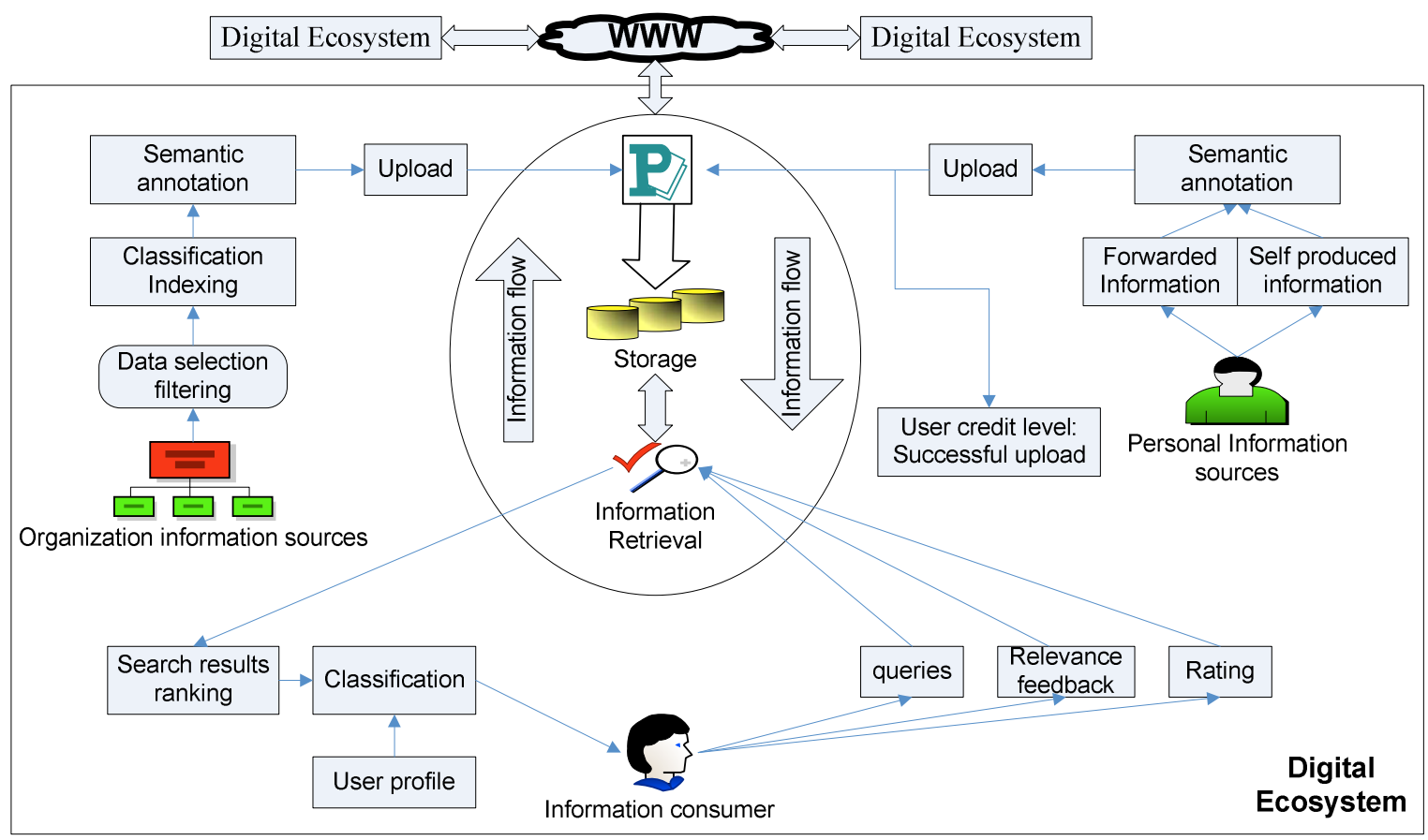

Fig.4 An intervention information flow model 
lection of $\mathrm{N}$ documents. It is usually calculated by $\log (\mathrm{N} / \mathrm{n})$. Normalization factor gives all documents, no matter how long or short are the documents, the same opportunity when calculating similarities between documents and queries. With the three factors, the tf-idf weighting is

$$
\mathrm{tf} \bullet \log (\mathrm{N} / \mathrm{n}) / \sqrt{\sum_{\text {vector }}\left(t f_{i} \bullet \log \left(N / n_{i}\right)\right)^{2}}
$$

Classification component groups the information items into a general predefined category hierarchy, such as the Yahoo! Web directory. The information publisher is responsible for the classification task to assure accurate classification results. The pre-processed information is then uploaded to the intranet of the digital ecosystem.

\section{2) Personal information sources}

Personal information includes forwarded information and self produced information. Forwarded information items are those which are forwarded directly to other users without any change to the original information. Self produced information items are those created by the user, or are modified information and delivered to the user by other users. All of the information also needs to be described before it is uploaded to the system. If the information item is successfully uploaded to the system, the user's credit level may be updated to have a higher privilege. User privilege is a policy which specifies users who have access to and control of sensitive and regulated information. Information published by high privilege user will be ranked higher when the information is retrieved by other users.

\section{3) Information retrieval}

The information retrieval component responds a user's search query by a list of ranked search results, which are the combination of the retrieved results from search engines, intranet, and commercial databases. The results are then classified based on a domain ontology, with consideration of user profiles. Users' relevance feedback is used to further refine the search results, and the users can also rate the presented results in terms of relevance and usefulness. Users are also allowed to add comments to the contents of the presented information.

Within the circle in Fig 4, over and above the information retrieval component, there are another two components: Publication and Storage. The Publication component accepts the uploaded information from different information sources, and then forwards the information to the Storage component, where the information is re-organized and indexed for the purposes of efficiency and effectiveness information retrieval.

\section{Description of the model}

Digital information is uploaded from two kinds of information sources: organization information sources and personal information sources. Organization information is firstly prepared and selected/filtered by the related authority/department in a digital ecosystem according the information security strategy of the organization. The selected information is then indexed and classified by the indexing and classification components. After providing the related meta data, semantic annotation component will check the meta data for the purpose of normalization of information publication. Only when the relevant meta data are provided and the related constraints are satisfied, can the information items be uploaded. A relevance weighting is automatically calculated for each of the key words in the keywords list. The first keyword is assigned the highest relevance weighting; the second is assigned the second highest relevance weighting, so on and so forth until the last keyword has the smallest relevance weighting.

As mentioned before, user information includes two types, forwarded information and self produced/modified information. Meta data are needed for the two information types. Before it can be uploaded, the information also needs to be checked and described by related meta data. After the information is successful uploaded, the user's credit file may be updated, and more privileges are granted to the user. In addition, if an uploaded information item is retrieved and evaluated by another user, the evaluation results are taken as a factor when search results are being ranked.

The information retrieval process is similar to that proposed by [14]. Users' personal information is collected from their personal computers; and data access privileges are assigned by the corresponding data security department [23]. The integrated information retrieval model retrieves information from different sources and the results are ranked and classified based on the created user profiles and a domain ontology. Part of the retrieved information is filtered according to users' access privileges. Implicit relevance feedback is combined with user profile to boost the precision of the returned search results. Further, users are allowed to comment or rate the quality of the given returned result.

The digital information flow model intends to govern the flow of information in digital ecosystems by controlling the quality of information sources via semantic annotation before an information item is uploaded to the system; consequently, controlling the quality of information sources improves the quality of the information retrieval service.

\section{FUTURE WORK AND DISCUSSION}

Information flow in digital ecosystems differentiates from the way the information has been transmitted in the past. Digital information now flows throughout information production, organization, access, and reproduction [7]. The ultimate purpose of increasing the quality of information flow, then, is to present relevant search results which can satisfy users' information needs. The intervention in information as described in the intervention information flow model (Fig. 4) is only one information flow governing strategy which can be effective in controlling the quality of the sources of information. In that regard, there are still issues to be addressed by other instruments and strategies.

First of all, the trade-off between efficiency and equality has not been addressed. The "Matthew Effect" ${ }^{2}$ of information usage improves the efficiency of the use of informa-

\footnotetext{
${ }^{2}$ The rich get richer and the poor get poorer.
} 
tion; and "Robin Hood effect" ${ }^{3}$ improves the free flow of information in a society, promotes equality in information use, and boosts universal access to information. The information asymmetry between the information rich countries and information poor countries is a well documented [24]; therefore any model that moves towards equality, in terms of information flow, and that maintains efficiency of the digital ecosystem, is moving towards the ideal.

Secondly, the realization of the ecological balance needs multilateral cooperation. Certain norms/standards need to be followed by information producers, storage providers, transmitters, and information consumers. We need to agree on a code of conduct and business rules [25]. A multilateral agreement is needed to set out the norms/standards for regulating the flow of information; these standards should be applicable throughout the life cycle of information flow. On this basis, an open, collaborative digital ecosystem can be established. The ecosystem would allow digital information to flow smoothly, and through the collaboration, members of the system can optimize knowledge sharing and rapid knowledge flow [25].

Finally, cultural influences are some other important factors needed not to be ignored in the digital ecosystem. Cultural infiltration and influence are enormous: not only can they affect the state of information flow - some kinds of information are positive and welcome in one area might be strictly forbidden, but culture can also affect information needs as well as the way information flows. These are areas that should not be ignored.

\section{Conclusion}

A number of information flow models have been proposed, each with their own characteristics, strengths and weaknesses. In this paper, an innovative information flow model is suggested from the perspective of information intervention, semantic annotation and governance.

The proposed model targets a collaborative system to improve the ability to control of information flow, and to provide refined search results to satisfy users' information needs. Comparing our model and other proposed models, our model pays more attention to operability and feasibility, and concentrates on how to improve the quality of information retrieval services by boosting the precision of retrieved results via personalization and classification.

\section{ACKNOWLEDGEMENT}

Thanks to Mr. David Packer for many valuable comments, suggestions, and advices.

\section{REFERENCES}

[1] H. Zhuge and X. Shi, "Toward the eco-Grid: A Harmoniously Evolved Interconnection Environment," Communications of the ACM, vol. 47, 2004, 79-83.

[2] A. L. Eryomin, "Information Ecology - a Viewpoint," International Journal of Environmental Studies, vol. 54, 1998, 241-253.

[3] M. Tungare, P. S. Pyla, Pérez-Quiñones., and S. Harrison, "Personal
Information Ecosystems and Implications for Design," The Computing Research Repository, vol. abs/cs/0612081, 2006

[4] Y. Wang, T. Wang, L. Tang, and D. Zhong, "Ecosystem Model Based Grid Resource Optimization Management," International of Computer Science and Network Security, vol. 7, 2007, 61-66.

[5] G. Lv, "Network Information ecological imbalance and Countermeasures," Information Research, vol. 121, 2007, 73-74.

[6] Q. Tang, "Internal irregularities of listed companies and the flow of information and obstruction," Modernization of Management, vol. 2006, 2006, 79-79.

[7] T. H. Davenport and L. Prusak, Information Ecology New York: Oxford University Press, 1997.

[8] B. A. Nardi and V. L. O'Day, Information Ecologies: Using Technology with Heart Cambridge, MA: MIT Press, 1999.

[9] K. S. Baker and G. G. Bowker, "Information ecology: open system environment for data, memories, and knowing," Journal of Intelligent Information Systems, vol. 29, 2007, 127-144.

[10] Y. Malhotra, "Information Ecology and Knowledge Management: Toward Knowledge Ecology for Hyper turbulent Organizational Environments.," in UNESCO Encyclopedia of Life Support Systems (EOLSS), D. L. Kiel, Ed. Paris: EOLSS Publisher, 2006.

[11] J. Fedorowicz, J. L. Gogan, A. W. Ray, and B. College, "The Ecology of Interorganizational Information Sharing," Journal of International Technology and Information Management, vol. 13, 2004, 7385.

[12] J. Martin, "CobiT: A Tool To Manage Information Ecology," Information Systems Control Jounal, vol. 3, 2003, 37-39.

[13] H. Boley and E. Chang, "Digital Ecosystems: Principles and Semantics," in Proceedings of the Inaugural IEEE Digital Ecosystems and Technologies Conference, 2007, 401-406.

[14] D. Zhu and H. Dreher, "An Integrating Text Retrieval Framework for Digital Ecosystems Paradigm," in Proceedings of the Inaugural IEEE Digital Ecosystems and Technologies Conference, 2007, 367-372.

[15] C. K. Work, "Information Flow \& the Institutional WWW", February 2, 2009, http://www.ukoln.ac.uk/webfocus/events/workshops/webmaster-jul1997/information-flow/iap$\underline{\mathrm{html}}$.

[16] J. Barwise and J. Seligman, Information Flow: the Logic of Distributed Systems Cambridge, UK: Cambridge University Press, 1997.

[17] G. Lowe, "Semantic Models for Information Flow," Theoretical Computer Science, vol. 315, 2004, 209-256.

[18] F. Drekske, Knowledge and the flow of information Stanford, CA: CSLI Publications, 1999.

[19] R. Heeks, "Communication Technology, Poverty and Development," Institute for Development Policy and Management, University of Manchester, Precinct Centre 1999.

[20] Y. Yang, "ICT and Information Flow Theory", February 1, 2009, http://papers.ssrn.com/sol3/papers.cfm?abstract id=613781.

[21] A. Kiryakov, B. Popov, D. Ognyanoff, D. Manov, A. Kirilov, and M. Goranov, "Semantic Annotation, Indexing, and Retrieval," in The SemanticWeb - ISWC 2003, D. Fensel, K. Sycara, and J. Mylopoulos, Eds. Berlin: Springer, 2003.

[22] G. G. Chowdhury, Introduction to Modern Information Retrieval, 2nd ed. London: Facet Publishing, 2004.

[23] D. Zhu and H. Dreher, "Personalized Information Retrieval in Digital Ecosystems," in Proceedings of the Second IEEE Digital Ecosystems and Technologies Conference, 2008, 580-585.

[24] R. Capurro, "Towards an Information Ecology," in Proceedings of a NORDINFO Seminar, Information Quality Definitions and Dimensions, 1990, 122-139.

[25] T. L. Friedman, The world is Flat London: Allen Lane-Penguin Group, 2005.

\footnotetext{
${ }^{3}$ An economic occurrence where income is redistributed so that economic inequality is reduced.
} 